\title{
Pronóstico precoz del tratamiento del cáncer cervical uterino por medio de la citología ${ }^{(*)}$
}

\author{
Profesor Pedro Nel Cardona, doctores Gustavo Isiaza Mejía \\ y Fernando Cardona Arango \\ Universidad de Antioquia. Medellín.
}

\section{Introducción}

El tratamiento del cáncer cervical uterino ha sido desde el descubrimiento de la actinoterapia enfocado hacia ella o hacia la cirugía, y desde aquellos tiempos hasta nuestros días se ha ciscutido la mayor efectividad de un tratamiento sobre el otro, sin haber llegado la oncología mundial a un acuerdo, siendo esta la razón por la cual encontramos que en unas escuelas prefieren la cirugía y en otras los tratamientos físicos ante un determinado grado clínico.

La causa de esto radica, en que en presencia de un mismo grado clínico, ambos métodos de terapia muestran éxito sólo en un determinado porcentaje, ignorándose cuáles enfermas van a quedar comprendidas entre las de curación radical.

Este problema, que tanto "Stress" causaba en el ambiente que rodeaba a la paciente con carcinoma cervical uterino, ha sido estudiado desde hace ya más de dos decenios, pero sólo en 1946 Smithers (1) presenta su estudio de biopsias seriadas post-tratamiento como indice pronóstico. Un año más tarde la doctora. Kuth Graham (2) presenta su trabajo sobre el efecto de las radiaciones en las células descamadas y su significado como pronóstico en cuanto a la curación definitiva; a este índice, la autora lo denominó R. R. (Radiación Response). En 1953 la doctora Graham publicó, en asociación con el doctor J. Graham (3), un artículo en donde estudiaba un determinado tipo de células descamadas y su importancia como índice de sensibilización a las radiacio-

(*) Trabajo presentado por los autores al VI Congreso Nacional de Medicina. Bogotá, 1959. 
nes; a este índice los autores denominaron S. R. (Sensitization Response). Este importante trabajo, con observaciones de más de cinco años, fue presentado por el doctor J. V. Meigs (4) en el Primer Congreso Mundial de Ginecología y Obstetricia, en Ginebra, en 1954.

Posteriormente, en 1957, la doctora Calderón de Laguna (1) publicó un trabajo en el cual hace un análisis de la persistencia de los signos citológicos de radiación y su relación con el pronóstico inmediato y tardío de la paciente; la autora denominó este índice R. P. (Radio Persistencia). Este índice R. P., y un estudio sobre el R. R. y S. R., fueron presentados como ponencia oficial en el Tercer Congreso Mexicano de Ginecología y Obstetricia (5).

\section{Definiciones del S. R., R. R. y R. P.}

Denomínase S. R. un fenómeno de respuesta de sensibilización, consistente en cambios citoplasmáticos en las células exfoliadas cérvico-vaginales, no cancerosas; estos cambios son: aumento de la densidad citoplasmática en las células basófilas, vacuolización del citoplasma y contorno citoplasmático bien delimitado. Estos cambios se observan principalmente en las células basales y parabasales, pero pueden también encontrarse en las células precornificadas, en las cuales pueden hallarse además como característica de $\mathbf{S}$. R. un doble núcleo (Figuras números $1,2,3$ у 4 ).

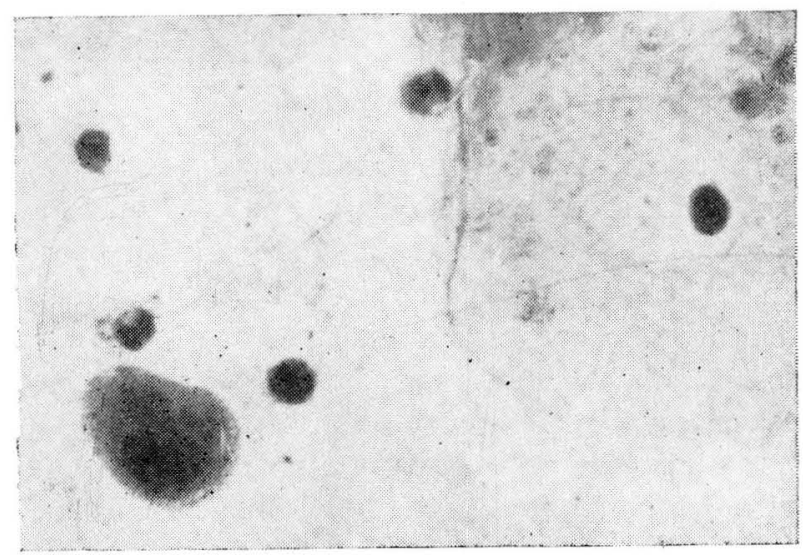

FIGUKA NUMERO 1 


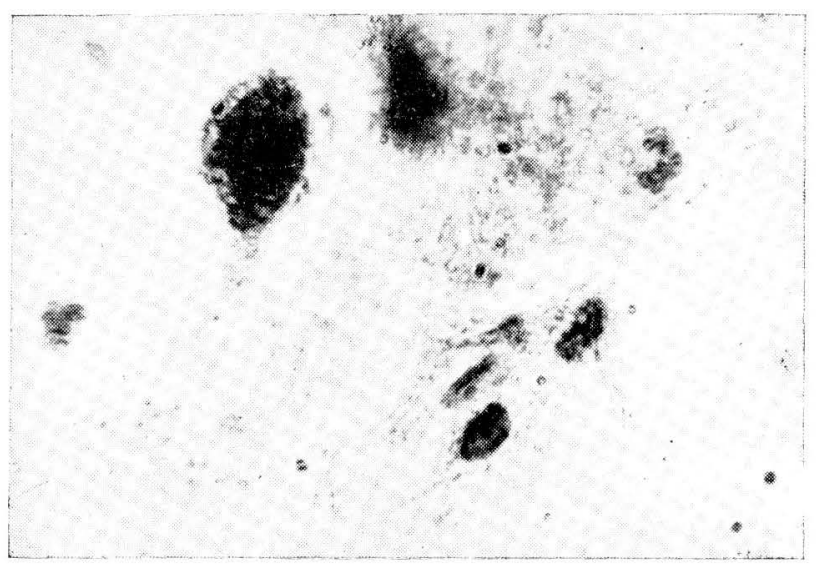

FIGURA NUMERO 2

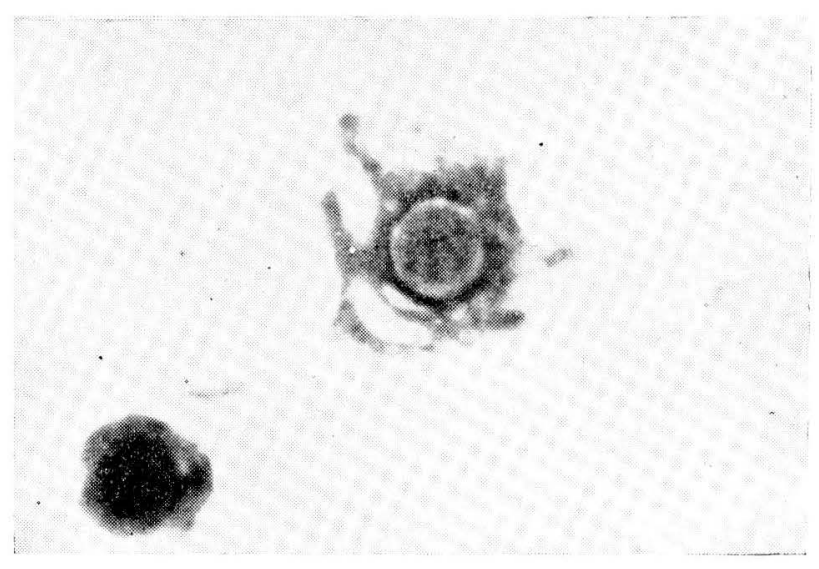

FIGURA NUMERO 3

Cuando estos cambios se encuentran en más de y\% de las células, se dice que el S. R. es bueno; cuando el porcentaje es menor de 9 , se dice que el $\mathbf{S}$. R. es pobre.

Denomínase $R$. $R$. a un fenómeno de radio-respuesta consistente en cambios de núcleo o del citoplasma en las células cxfoliadas cérvico-vaginales, no cancerosas; estos cambios son: 


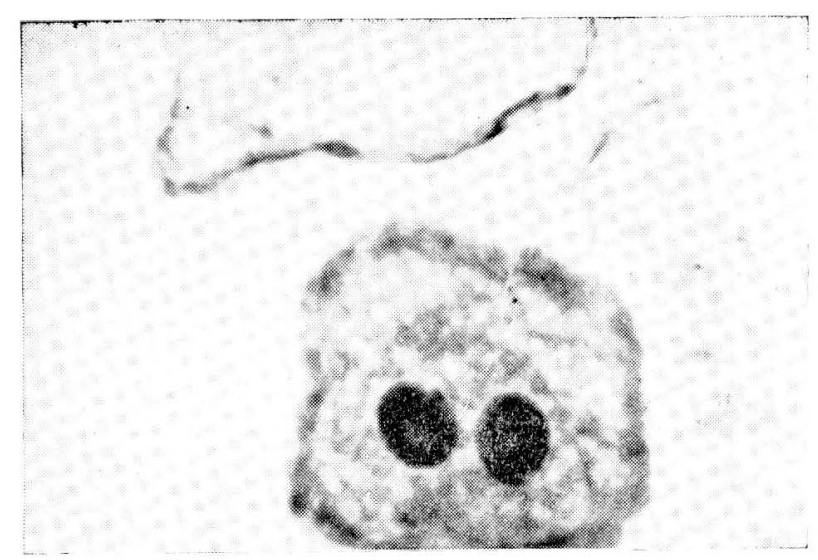

FIGURA NUMERO 4

un aumento del citoplasma, contorno irregular y morfología abigarrada citoplasmática, presencia de vacuolas de pequeño o grancie diámetro intracitoplasmáticas, aumento del tamaño celular tanto del citoplasma como del núcleo, arrugamiento del núcleo como si la membrana nuclear fuera más grande que su conterido, distribución en grumos de la cromatina en células con amplio protoplasma, vacuolización intranuclear y dos o más núcleos (Figuras números 5, 6, 7, 8, 9, 10, 11 y 12).

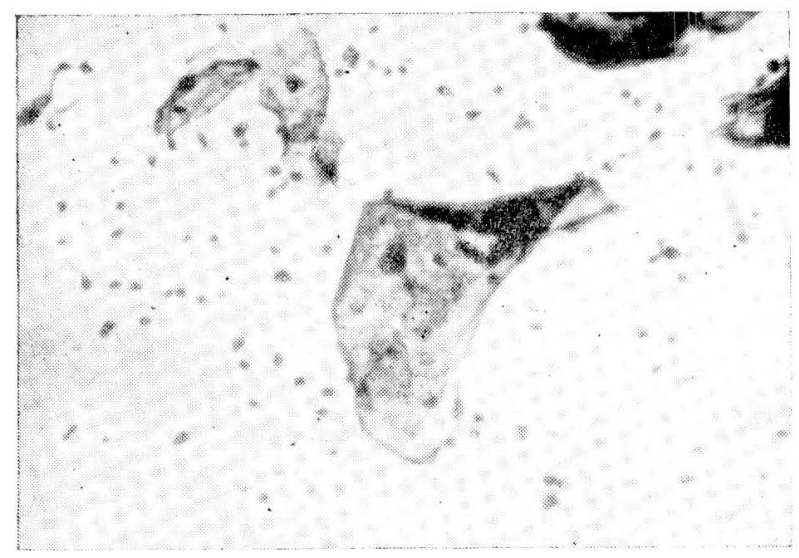

FIGURA NUMERO 5 


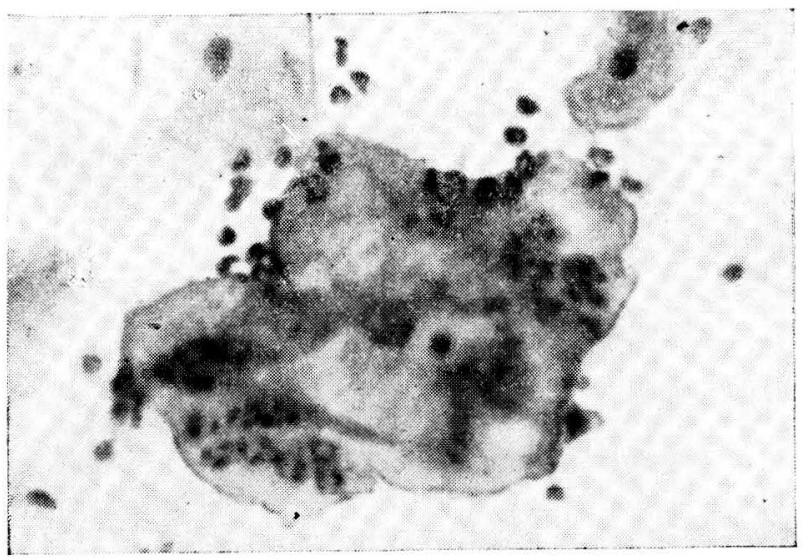

FIGURA NUMERO 6

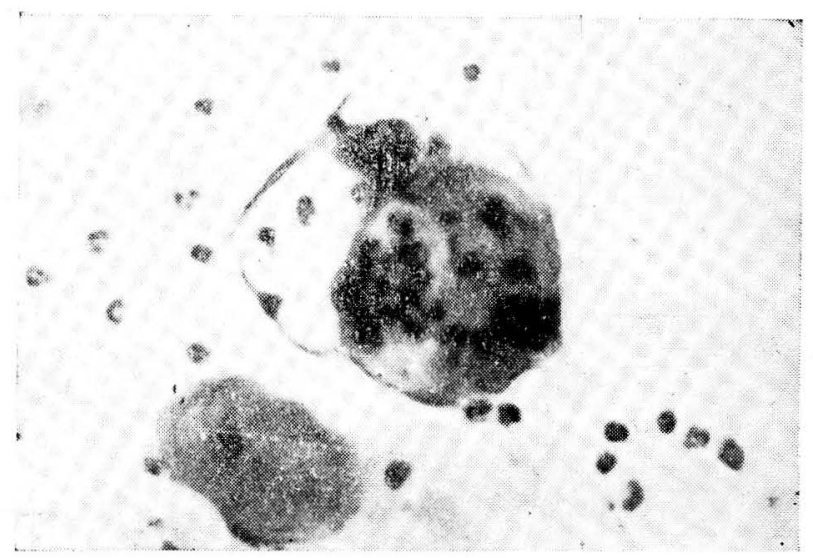

FIGURA NUMERO 7 


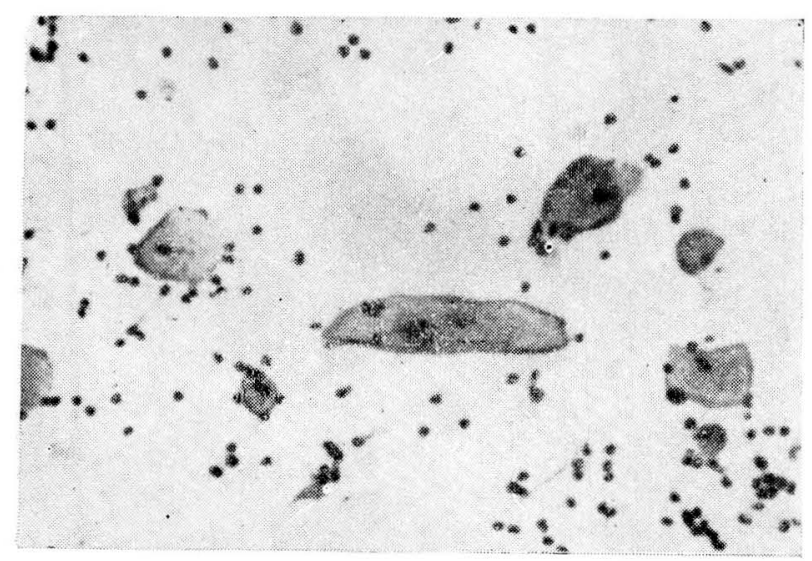

FIGURA NUMERO 8

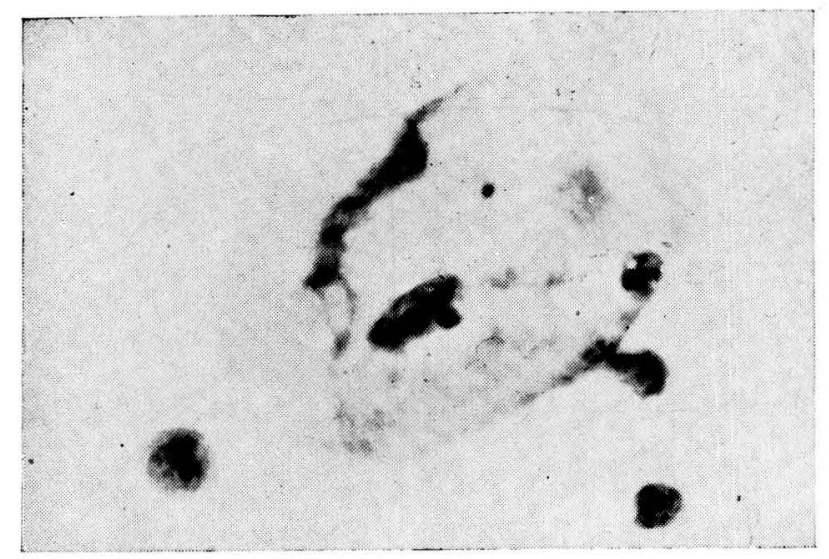

FIGURA NUMERO 9 


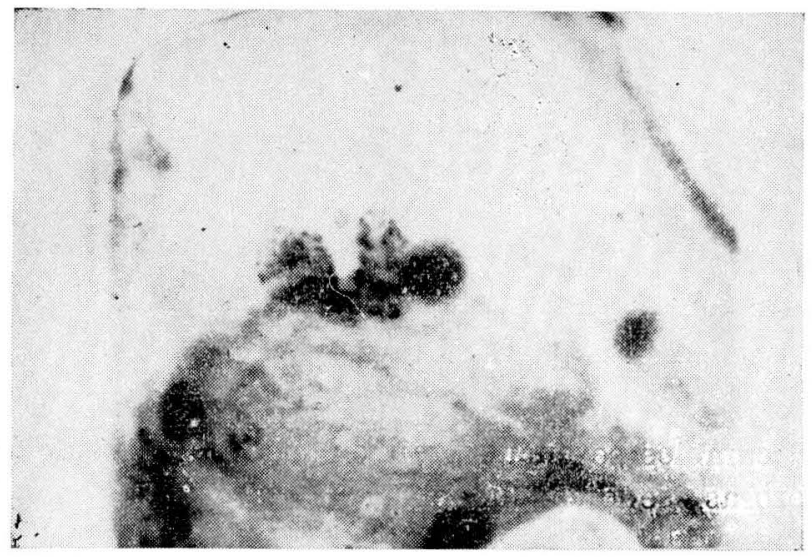

FIGURA NUMERO 10

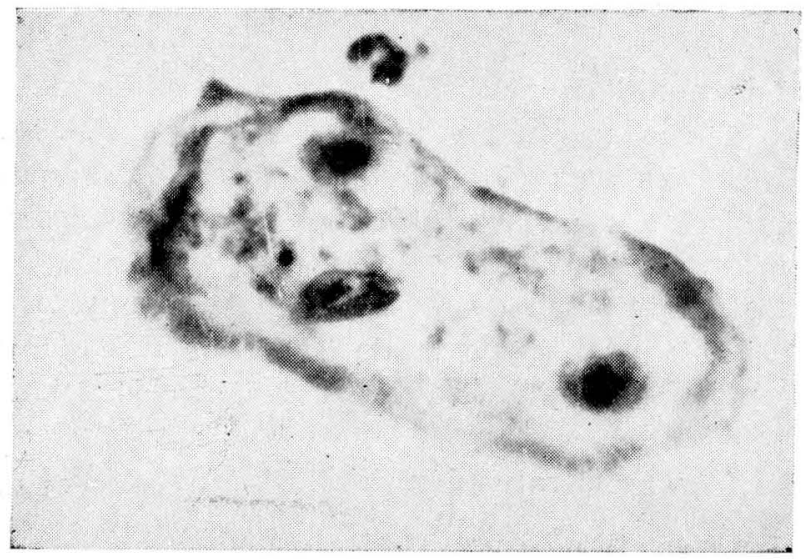

FIGURA NUMERO 11 


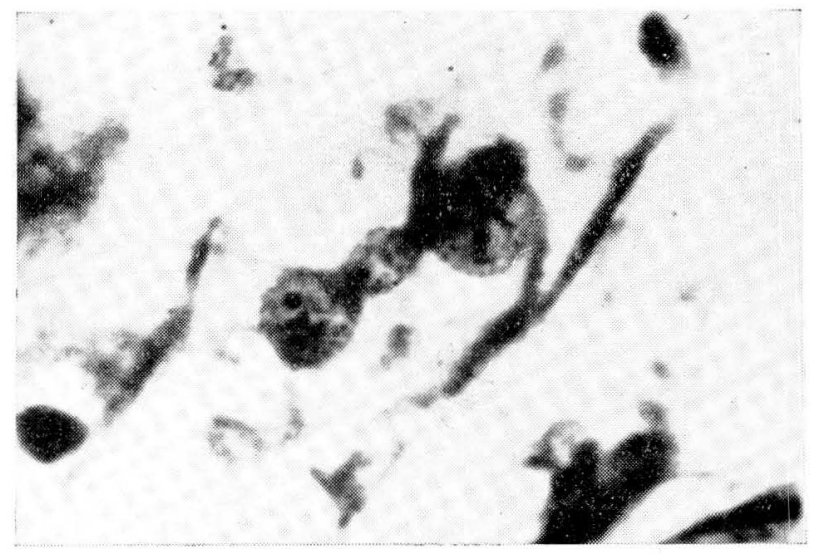

FIGURA NUMERO 12

Cuando estos cambios se encuentran en más del $75 \%$ de las células no cancerosas, se dice que el R. R. es bueno; cuando el porcentaje es menor del $65 \%$, se dice que el $R$. $R$. es malo.

Denomínase R. P. a un fenómeno de persistencia en las células' exfoliadas de los cambios producidos por la actinoterapia; por consiguiente, los cambios citológicos son los ya descritos en el $R$. R. y con este método no hacemos más que observar la duración, o sea la persistencia, de dichos cambios en las células descamadas a partir de la iniciación del tratamiento actínico. En el R. P. no se tiene en cuenta el porcentaje.

\section{Técnica}

Las muestras para hacer los extendidos deben tomarse del fondo de saco vaginal posterior, ya sea para S. R., R. R. o R. P., pues éste ha sido el procedimiento empleado por los autores y los indices por ellos establecidos están basados en la toma del fondo de saco vaginal posterior.

Para la tinción del extendido puede emplearse la coloración de Papanicolaou (6) o la de Shorr modificada (7). En nuestra concepto es más práctica y efectiva la coloración de Shorr.

\section{Evaluación del S. R., S. R. y R. P.}

La efectividad como índice pronóstico del S. R. y su utilidad para la elección del tratamiento quirúrgico o actínico nos lo 
enseñan la doctora R. Graham, el doctor J. B. Graham y la doctora Winifred (8) con sus estadísticas, en donde con S. R. bueno, $73 \%$ de las pacientes sobreviven más de 5 años después del tratamiento actínico y con S. R. pobre, solo $18 \%$ sobreviven después de 5 años del tratamiento actínico (Cuadro número 1).

CUADRO NUMERO 1

Tratamiento actínico.

Supervivencia de 5 años.

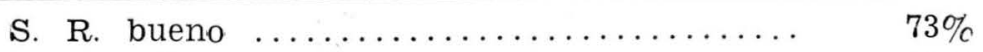

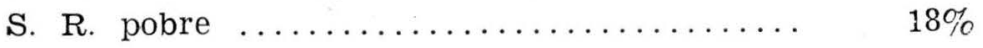

Surg. Gynec. and Obst.

Volumen 99, 555. 1954.

En pacientes tratadas con histerectomía radical $\mathrm{y}$ linfadenectomía regional, las estadísticas de los Graham y winifred (8) nos indican que con S. R. bueno, 39\% de las pacientes sokreviven más de 5 años y con S. R. pobre, $71 \%$ de las pacientes sobreviven después del tratamiento quirúrgico más de un lustro. (Cuadro número 2).

CUADRO NUMERO 2

\section{Tratamiento quirúrgico.}

Supervivencia de 5 años.

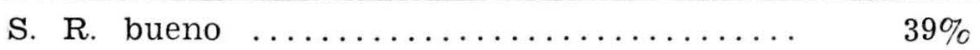

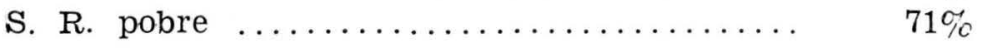

Surg. Gynec. and Obst.

Volumen 99, 555. 1954.

La efectividad del R. R. como índice pronóstico nos la presentan los doctores Graham y Graham (9) con sus estadísticas, en donde las pacientes con $R$. R. bueno muestran una supervivencia mayor de 5 años en un $65 \%$ y con $R$. $R$. malo, sólo sobreviven a los 5 años un $8 \%$ (Cuadro número 3 ). 


\section{CUADRO NUMERO 3}

\section{R. R. y supervivencia}

mayor de 5 años.

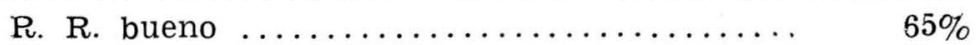

R. R. malo $\ldots \ldots \ldots \ldots \ldots \ldots \ldots \ldots \ldots \ldots \ldots \ldots$

Cáncer. Volumen 8, 55. 1955.

La efectividad del $R$. P. como índice pronóstico nos la expresa la doctora Calderón de Laguna (1) con sus estadísticas, en donde con R. P. de 7 meses o más, la supervivencia a los 5 años As del $95 \%$ y con R. P. menor de 6 meses, sólo un $26 \%$ sobrevivieron 5 años; además, con $R$. P. de 11 o más meses, la supervivencia a los 5 años es del 100\% (Cuadro número 4).

\section{CUADRO NUMERO 4}

\section{R. P. y supervivencia} mayor de 5 años.

R. P. de 11 o más meses $\ldots \ldots \ldots \ldots \ldots \ldots \ldots$ 10. $\%$

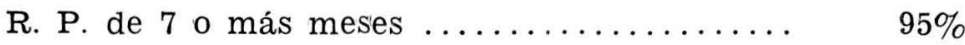

R. P. de 6 o menos meses ............. $26 \%$

Rev. Inst. Nal. Cancerol. México.

Volumen I, 218. 1957.

\section{Comentario}

Nosotros en la cátedra de ginecología de la Facultad de Medicina de la Universidad de Anitioquia, estamos en la actualidad clefiniendo la conducta terapéutica por el estudio del S. R. y estamos haciendo el R. R. y el R. P., y esperamos exponer en posterior comunicación nuestra estadística con sus conclusiones cuando nos lo permitan los factores tiempo y número de casos.

No obstante, juzgamos oportuno presentar en forma sintética la Radio Respuesta en 50 de nuestras pacientes con $\mathbf{S}$. $R$. previo. A las enfermas portadoras de un S. R. pobre, se les aplicó 150 miligramos de testosterona basados en la ponencia sobre este tema presentada en el Tercer Congreso Mexicano de Obstetricia y Ginecología (5) (Cuadro número 5). 
CUADRO NUMERO 5

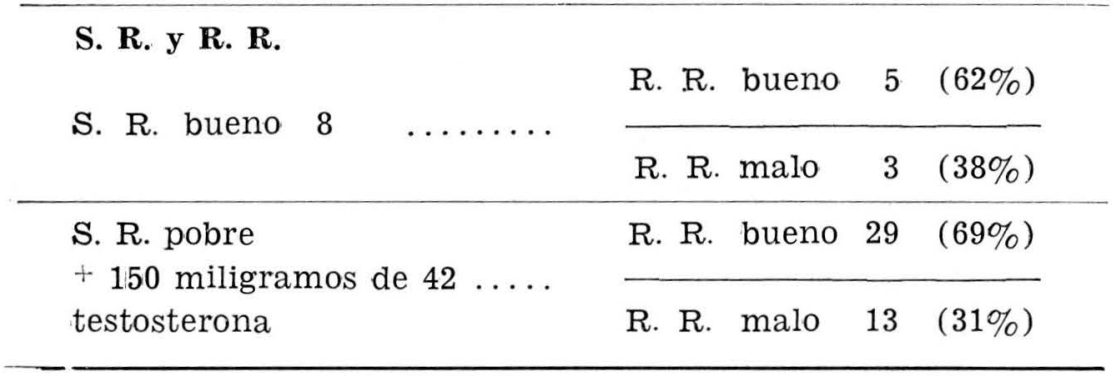

Vemos en el cuadro número 5 que el $\mathbf{S}$. R. es un orientador del pronóstico, pero no en forma absoluta, ya que solo obtuvimos en 5 de nuestras enfermas un $R$. R. bueno $(62 \%)$, y en 3 de ellas un R. R. malo (38\%), del total de 8 pacientes con S. R. bueno.

Podemos apreciar también en el cuadro número 5 que en 42 pacientes con $\mathbf{S}$. R. pobre tratadas con 150 miligramos de testosterona, obtuvimos un R. R. bueno en 29 de ellas (69\%), y un R. R. malo en solo 13 enfermas (31\%).

Los autores mexicanos (5), en casos con $S$. R. pobre que no recibieron testosterona, encontraron un $R$. $R$. bueno en $22 \%$, y nosotros, en pacientes con S. R. pobre a quienes administramos 150 miligramos de testosterona, encontramos un $R$. $R$. bueno en $69 \%$.

La comparación de las dos cifras anteriores nos muestran un $47 \%$ de mayor Radio Respuesta buena en pacientes que estuvieron sometidas al tratamiento androgénico, lo que prueba la gran influencia de este factor en el pronóstico del carcinoma cervical uterino, y ratifica las experiencias realizadas en el Hospital General de México, D. F.

Estamos íntimamente convencidos del criterio de credibilidad del S. R., R. R. y R. P., y de su gran utilidad práctica, y ésta es la razón por la cual insistimos en su importancia en la II Convención Nacional de Obstetricia y Ginecología celebrada en Cali en 1955 (10), y en posterior artículo de "Antioquia Médica" (11).

Esperamos que la presente comunicación expuesta ante el VI Congreso Médico Nacional, sirva de base para que en un futuro próximo en todos los centros oncológicos colombianos, la 
conducta terapéutica y el estudio del pronóstico del cáncer cervical uterino se rijan por los cambios anotados en la citología exfoliativa.

\section{Resumen}

Se revisan los métodos actuales de citología exfoliativa para el tratamiento y pronóstico precoz del cáncer cervical uterino y se demuestra su valor. La suma de todas estas técnicas define la conducta terapéutica y predice la evolución del neo del cérvix.

Carrera 46, número 52-95, MEDELLIN.

\section{BIBLIOGRAFIA}

1. LAGUNA J. C. de.-Persistencia de los signos citológicos de radiación R. P. como dato pronóstico en el carcinoma cérvico-uterino tratado por radiaciones. Rev. Inst. Nal. Cancerol. México. Volumen I, 218. 1959.

2. GRAHAM R. M.-The effect of radiation on vaginal cells in cervical carcinoma. I. Description of cellular changes. II. The pronostic significance. Surg. Gynec. Obst. Volmen 84, 153. 1957.

3. GRAHAM R. M. y GRAHAM J. B.-A cellular index of sensitivity to ionizing radiation; the sensitization response. Cancer. 6: 215, 1953.

4. MEIGS J. V.-The treatment of early carcinoma of the cervix. Can results be improved? La Prophylaxie en Gynécologie et Obstetrique. Georg. Genéve. página 588. 1954.

5. GARCiA G., GRAham J. C., LAGUNA J. C. de, MUNGUiA H., URRUTIA M.-Importancia de los signos R S. (Radio Sensibilidad) y R. R. (Radio Respuesta) en el pronóstico y tratamiento del carcinoma cérvico-uterino. Memoria de los Terceros Congresos Latinoamericanos y Mexicano de Obstetricia y Ginecología. 283, 1958.

6. PAPANICOLAOU G. N.-Atlas of Exfoliative Cytology. Harvard University Press, Cambridge, Mass. Página 3, 1954.

7. PUNDEL J. P.-Acquisitions récentes en Cytologie Vaginale Hormonale. Masson y Cía. Ed. Página 15. 1957.

8. GRAHAM R. M., GRAHAM J. B. y WINIFRED L.-Prognosis in cancer of the uterine cervix based on the vaginal smear before treatment. S. R. the sensitization response. Surg. Gynec. and Obst. Volumen 99, 555. 1954.

9. GRAHAM M. R. y GRAHAM J. B.-Cytological prognosis in cance: of the uterine cervix treated radiologically. Cancer. Volumen 8, 55. 1955.

10. CARDONA P. N., DIAZ C. R., ISAZA G.-Tratamiento del carcinoma uterino. Memorias de la II Convención Colombiana de Obstetricia : Ginecología. Cali. Edición Especial de la "Revista Colombiana de Obstetricia y Ginecología”. Bogotá. Página 119. 1955.

11. CARDONA F.-Importancia de la Coloración de Papanicolaou en el đaiagnóstico y pronóstico de las ginernnatías. "Antioquia Médica". Volumen VII, 171. 1957. 